\title{
Numerical Study of the Hydrodynamic Performance of Two Wiggling Hydrofoils in Diagonal Arrangement
}

\author{
Xingjian Lin'1, Guoyi He", Xinyi He², Qi Wang1, Longsheng Chen ${ }^{1}$ \\ ${ }^{1}$ The School of Aircraft Engineering, Nanchang Hangkong University, Nanchang, China \\ ${ }^{2}$ The School of Software, Nanchang Hangkong University, Nanchang, China \\ Email: 850296072@qq.com
}

How to cite this paper: Lin, X.J., He, G.Y., He, X.Y., Wang, Q. and Chen, L.S. (2017) Numerical Study of the Hydrodynamic Performance of Two Wiggling Hydrofoils in Diagonal Arrangement. Journal of Applied Mathematics and Physics, 5, 31-38. http://dx.doi.org/10.4236/jamp.2017.51005

Received: November 18, 2016 Accepted: December 28, 2016 Published: January 4, 2017

\begin{abstract}
The hydrodynamics in fish school are studied through simulating the flow over two wiggling hydrofoils in diagonal arrangement by using the immersed boundary method, both in-phase and anti-phase wiggling motions are considered in this study, the effect of the longitudinal spacing on the hydrodynamic performance has been investigated. It is revealed that, when the longitudinal spacing is smaller than body length, the diagonal formation is helpful to the follower but harmful to the leader; when the longitudinal spacing is larger than body length, the opposite effect is occurring; and a significant enhancement of the propulsive performance is obtained when the longitudinal spacing is optimized.
\end{abstract}

\section{Keywords}

Hydrodynamic Performance, Fish School, Diagonal Arrangement, Longitudinal Spacing

\section{Introduction}

The diamond performance of fish school has been studied by biologists and physicists for several decades [1] [2]. The hydrodynamic interaction between flow and fish is the crucial question for understanding the intrinsic mechanism in fish school, since fish can swim efficiently through the active and passive responses to the hydrodynamic reaction [3] [4]. The passive flexible filaments are also used to investigate the passive responses to the fluid-structure interaction in fish school, for two paratactic filaments, two flapping states, in-phase flapping state and out-of-phase flapping state, have been observed experimentally [5] and numerically [6], which means that the hydrodynamic performance relates to the 
arrangement [7], for two tandem filaments, a drag increase has been observed for the follower while the leader was unaffected [8] [9] [10]. To understand the active responses to the hydrodynamic reaction in fish school, the active wiggling hydrofoils have been used in the previous studies, for two tandem hydrofoils, a significant augmentation of the thrust has been obtained by the leader, while the follower experienced the decreased thrust [11] [12], for two paratactic hydrofoils, the increased drag and decreased power were observed when wiggled in-phase, but the increased drag and expensive power consumption were found when wiggled anti-phase [13]. The diagonal formation, which combines the tandem and paratactic formations, is a ubiquitous arrangement in fish school, investigating the hydrodynamic performance of two wiggling hydrofoils in diagonal formation is worthwhile for a complete understanding of the intrinsic mechanism in fish school, although some understanding has been obtained through the tandem and paratactic models, this is the motivation of the current work.

In this study, the flow over two wiggling hydrofoils in diagonal arrangement has been simulated using immersed boundary method [14], the thrust coefficient, the power coefficient, the propulsive efficiency and the vortex structure are analyzed. The rest of this paper is organized as follows. Section 2 presents the physical model and numerically method. Section 3 addresses the simulation results in detail with discussions. Finally, some conclusions are drawn in Section 4.

\section{Physical Model and Numerically Method}

In this paper, we consider the hydrodynamic interactions between two diagonal hydrofoils in a two-dimensional incompressible viscous flow, as shown in Figure 1, a NACA0012 airfoil is used as the contour of the hydrofoils, the wiggling motion is described as follow:

$$
\begin{gathered}
\mathrm{y}_{0}\left(\mathrm{x}_{1}, \mathrm{t}\right)=\mathrm{A}\left(\mathrm{x}_{1}\right) \cos \frac{2 \pi}{\lambda}\left(\mathrm{x}_{1}-\mathrm{ct}\right), \\
A\left(x_{1}\right)=a_{0}-a_{1} x_{1}+a_{2} x_{1}^{2} .\left(0 \leq x_{1} \leq 1\right)
\end{gathered}
$$

where $y_{0}$ is the lateral coordinate of the midline along the hydrofoil, $\lambda$ is the wavelength, $\mathrm{c}$ is the phase velocity, $\mathrm{x} 1$ is defined as $x_{1}=\left(x-x_{0}\right) / L, x_{0}$ is the position of the leading edge of hydrofoil, $\mathrm{L}$ is the length of the hydrofoil, $A\left(x_{1}\right)$ is the amplitude along the hydrofoil, coefficients $a_{0}=0.02, a_{1}=0.0825, a_{2}=0.1625$, are

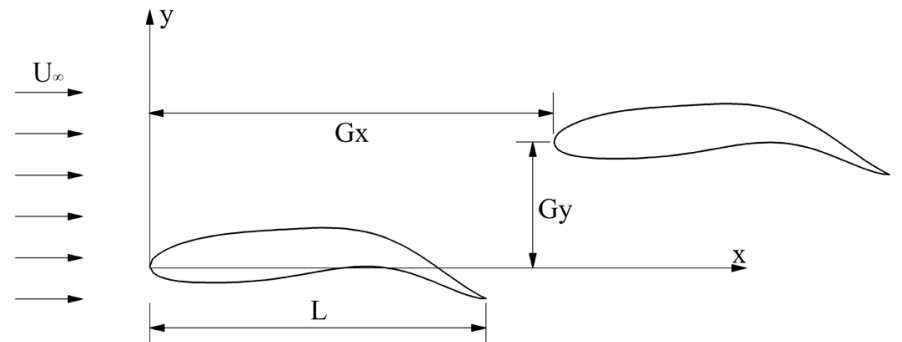

Figure 1. Schematic views of mathematic model of two hydrofoils arranged in diagonal formation, $U_{0}$ : the Initial velocity of the flow, $L$ : the length of the hydrofoil, $G_{X}$ and $G_{\dot{r}}$ the longitudinal and lateral spacing between two hydrofoils. 
calculated from the data of steadily swimming motion of fish [13], the period $\mathrm{T}$ of the wiggling motion is determined by $\lambda$ and $c, T=\lambda / c$. The dimensionless separations are defined as follows: the longitudinal spacing $D_{x}=G_{x} / L$, the lateral spacing $\mathrm{D}_{\mathrm{y}}=\mathrm{G}_{\mathrm{y}} / \mathrm{L}$.

The governing equations of a two-dimensional incompressible viscous flow are written as follows:

$$
\begin{gathered}
\frac{\partial u}{\partial \mathrm{t}}+\nabla \cdot(u u)=-\nabla \mathrm{p}+\frac{1}{\operatorname{Re}} \nabla^{2} u+f \\
\nabla \cdot u=0
\end{gathered}
$$

where $\boldsymbol{u}$ is the velocity, $p$ is the pressure, the Reynolds number $\operatorname{Re}=L U_{0} / v, v$ is the viscosity, and $\boldsymbol{f}$ is the Eulerian force density. A simple immersed boundary method [14] is employed to calculate the interacting force between the hydrofoil and the fluid, the complete details of the algorithm and the validations are available in the previous work [15].

The time-averaged thrust coefficient, the time-averaged power coefficient and propulsive efficiency for each hydrofoil are calculated as follows:

$$
\overline{\mathrm{C}}_{\mathrm{t}}=\frac{1}{\mathrm{~T}} \int_{\mathrm{t}}^{\mathrm{t}+\mathrm{T}} \int \frac{-F_{1}}{\rho \mathrm{U}_{0}^{2} \mathrm{~L} / 2} \mathrm{dsdt}, \overline{\mathrm{C}}_{\mathrm{w}}=\frac{1}{\mathrm{~T}} \int_{\mathrm{t}}^{\mathrm{t}+\mathrm{T}} \int \frac{-F_{2}\left(\partial \mathrm{y}_{0} / \partial \mathrm{t}\right)}{\rho \mathrm{U}_{0}^{2} \mathrm{~L} / 2} \mathrm{dsdt}, \eta=\frac{\overline{\mathrm{C}}_{\mathrm{t}}}{\overline{\mathrm{C}}_{\mathrm{w}}}
$$

where $F_{1}$ and $F_{2}$ are the x-component and y-component of the Lagrangian force, respectively.

For the whole two-hydrofoil system, the coefficients and efficiency are defined as follows:

$$
\overline{\mathrm{C}}_{\mathrm{t} 0}=\left(\overline{\mathrm{C}}_{\mathrm{t} 1}+\overline{\mathrm{C}}_{\mathrm{t} 2}\right) / 2, \quad \overline{\mathrm{C}}_{\mathrm{w} 0}=\left(\overline{\mathrm{C}}_{\mathrm{w} 1}+\overline{\mathrm{C}}_{\mathrm{w} 2}\right) / 2, \quad \eta_{0}=\overline{\mathrm{C}}_{\mathrm{t} 0} / \overline{\mathrm{C}}_{\mathrm{w} 0}
$$

where $\bar{C}_{t 1}, \bar{C}_{t 2}, \bar{C}_{w 1}, \bar{C}_{w 2}$ are the coefficients for each hydrofoil, respectively.

\section{Results and Discussion}

The parameters in the current work are defined as follows: $\mathrm{U}_{0}=1.0, L=1.0, \mathrm{Re}$ $=200.0$. To generate the reversed Von-Kármán vortices and thrust, the wiggling parameters are set as $\lambda=1.0, c=5.0$, both in-phase and anti-phase motions are considered. The separation distances are set as: $\mathrm{D}_{\mathrm{x}}=0.0-3.0, \mathrm{D}_{\mathrm{y}}=0.3-0.4$, the values of lateral spacing selected here are similar to the optimal distance in the theory of Weihs [1], and the effect of the longitudinal spacing on the hydrodynamic performance are analyzed typically.

\subsection{In-Phase Wiggling Hydrofoil}

The performance of in-phase wiggling hydrofoils, such as the thrust coefficient, the power coefficient and the propulsive efficiency, has been shown in Figure 2. It is indicated that, when the longitudinal spacing is smaller than the body length $\left(D_{x}<1.0\right)$, the downstream hydrofoil has a significant enhancement of the thrust coefficient, but the upstream hydrofoil has the reduction, compared to the single hydrofoil, and so as the propulsive efficiency, although the time-averaged power coefficient of the hydrofoils is larger than that of single foil in some cases. This 

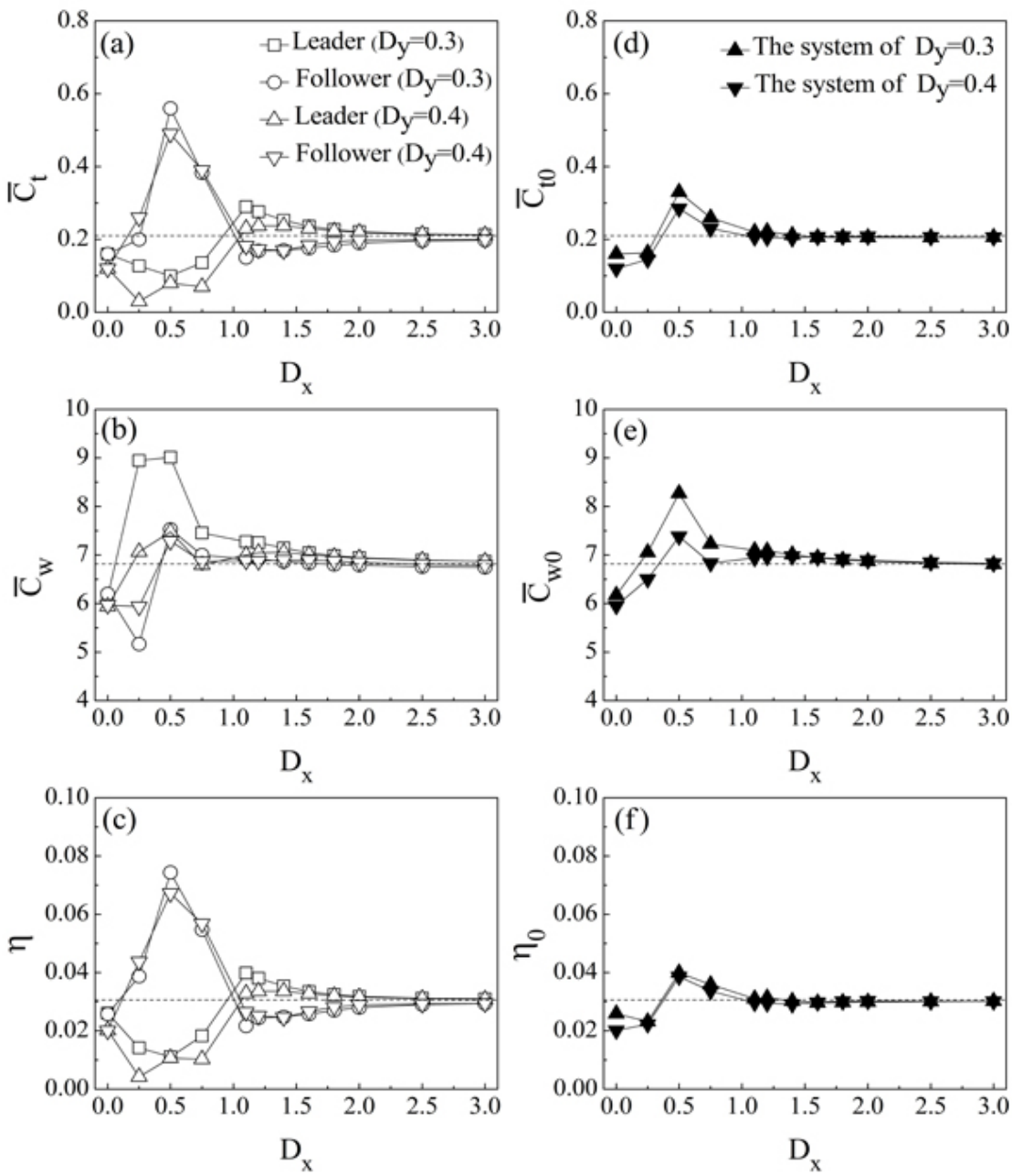

Figure 2. The time-averaged thrust coefficient for (a): each hydrofoils and (d): the whole system; The time-averaged power coefficient for (b): each hydrofoils and (e): the whole system; The propulsive efficiency for (c): each hydrofoils and (f): the whole system. The empty symbols of square and circle denote the variables of the leader and the follower in the diagonal formation of $D_{y}=0.3$, respectively. The empty symbols of triangle and inverted triangle denote the variables of the leader and the follower in the diagonal formation of $\mathrm{D}_{\mathrm{y}}=0.4$, respectively. The filled symbols of triangle and inverted triangle denote the variables of the whole two-hydrofoil system of $\mathrm{D}_{\mathrm{y}}=0.3$ and $\mathrm{D}_{\mathrm{y}}=0.4$, respectively. The dashed lines denote the values of each propulsive variables of a single hydrofoil.

may arise because the vortices generated by the leader are captured by the follower, and merged into the wake of the follower, as shown in Figure 3. Moreover, if two hydrofoils are arranged in side-by side formation $\left(D_{x}=0.0\right)$, the thrust coefficients for each hydrofoils are smaller than that of a single hydrofoil, and the wake is weak, as shown in Figure 4(a), it is similar to the previous result that the paratactic hydrofoils experienced increased drag [13]. When the longitudinal spacing is larger than body length $\left(D_{x}>1.0\right)$, the thrust coefficient of the upstream body is larger than that of the downstream body, and so as the propulsive efficiency and the time-averaged power coefficient, since the follower has experienced the jet flow produced by the leader, as shown in Figure 4(b). 


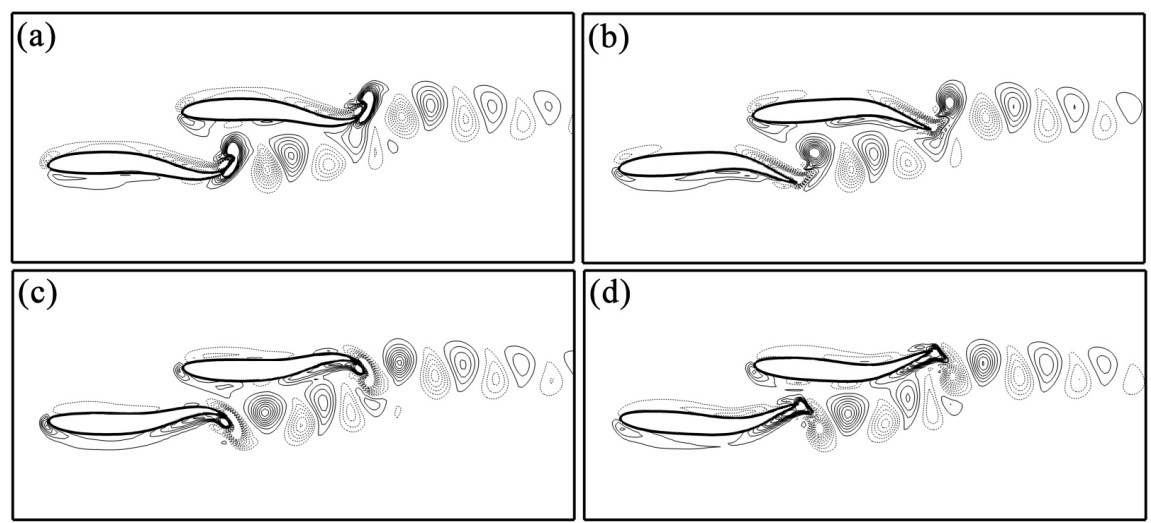

Figure 3. Vorticity contours over one period in the in-phase case of $D_{x}=0.75, D_{y}=0.3$, (a) $0.3 \mathrm{~T}$; (b) $0.5 \mathrm{~T}$; (c) $0.8 \mathrm{~T}$; (d) $1.0 \mathrm{~T}$. The vorticity value ranges from -90 to 90 with intervals of 10 , solid and dashed lines denote positive and negative contours.
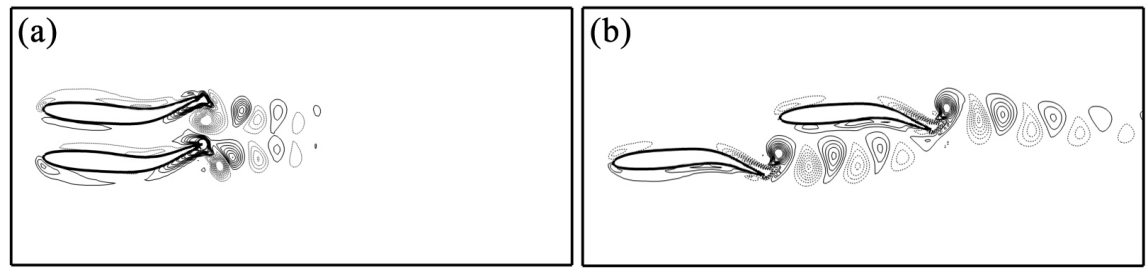

Figure 4. Vorticity contours in the in-phase case of (a) $D_{x}=0.0, D_{y}=0.3 ;(b) D_{x}=1.1 ; D_{y}$ $=0.3$. The vorticity value ranges from -90 to 90 with intervals of 10 , solid and dashed lines denote positive and negative contours.

For the whole in-phase system, as shown in Figures 2(d)-(f), the thrust coefficient and propulsive efficiency are smaller than that of a single hydrofoil when $D_{x}=0.0,0.25$, but larger when $D_{x}=0.5,0.75$. The best propulsive efficiency is obtained when $\mathrm{D}_{\mathrm{x}}=0.5$, although the more power is required. When the longitudinal spacing is larger than body length $\left(D_{x}>1.0\right)$, the propulsive performance of the whole two-hydrofoil system is similar to that of a single hydrofoil.

\subsection{Anti-Phase Wiggling Hydrofoils}

The propulsive performance of anti-phase wiggling hydrofoils has been shown in Figure 5. It is revealed that, both the thrust coefficient and the propulsive efficiency for the downstream hydrofoil is higher than that for the upstream hydrofoil when the longitudinal spacing is smaller than the body length $\left(D_{x}<1.0\right)$, since the vortices generated by the former are also captured and merged into the wake of the latter, as shown in Figure 6. Moreover, the hydrodynamic performance for two paratactic hydrofoils $\left(D_{x}=0.0\right)$ are better than that for a single one, and a strong wake is generated by the paratactic system, as shown in Figure $7(a)$. When the longitudinal spacing is larger than body length $\left(D_{x}>1.0\right)$, the propulsive performance of the upstream hydrofoil is better than that of the downstream hydrofoil, since the downstream body has experienced the jet flow produced by upstream one, as shown in Figure 7(b).

For the whole anti-phase system, a significant enhancement of thrust coefficient has been obtained when $D_{x}=0.0,0.25$, compared to a single hydrofoil, and 

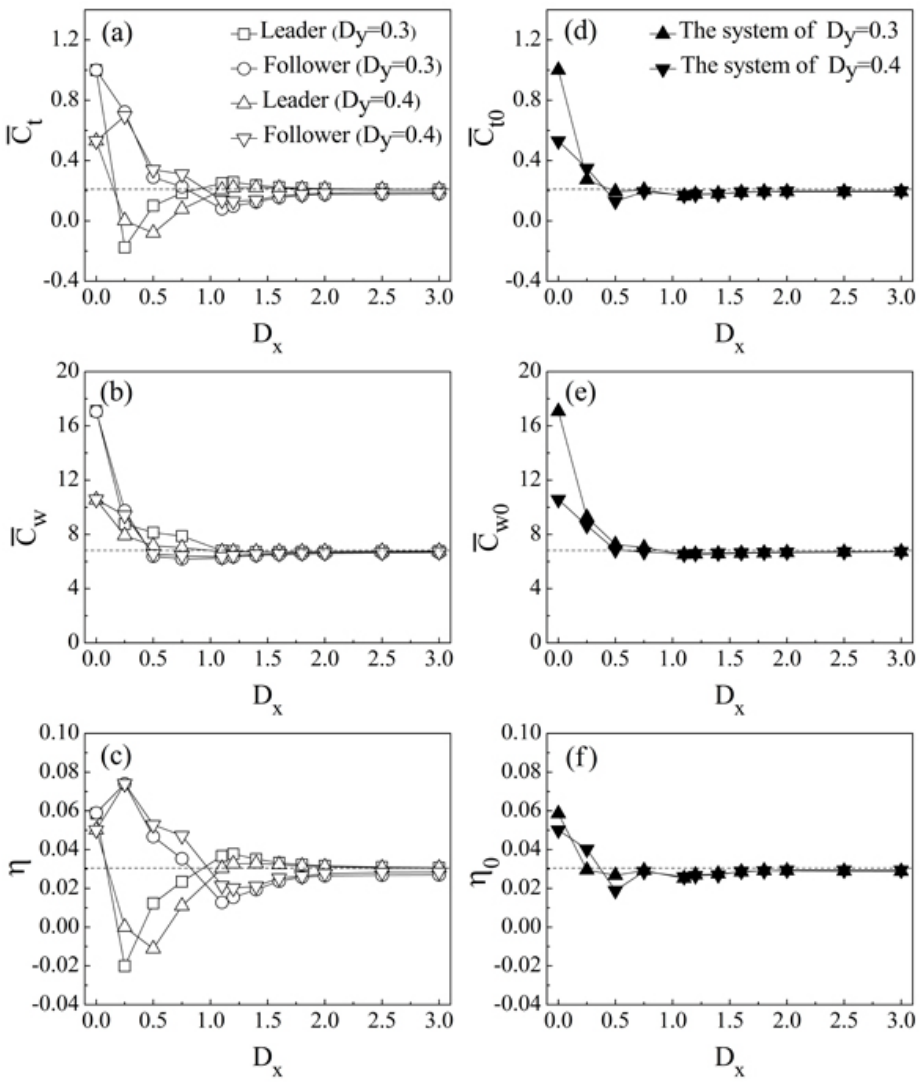

Figure 5. The time-averaged thrust coefficient for (a): each hydrofoils and (d): the whole system; the time-averaged power coefficient for (b): each hydrofoils and (e): the whole system; and the propulsive efficiency for (c): each hydrofoils and (f): the whole system. Refer to Figure 2 for the meaning of symbols.

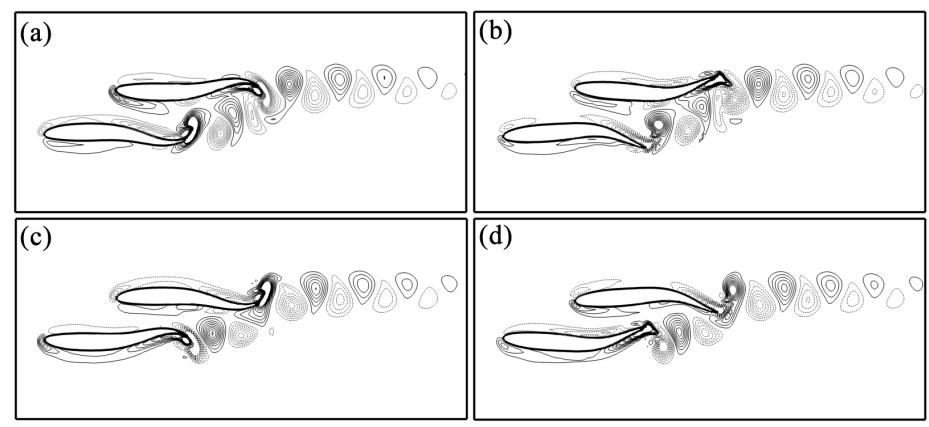

Figure 6. Vorticity contours over one period in the anti-phase case of $D_{x}=0.5, D_{y}=0.3$, (a) $0.3 \mathrm{~T}$; (b) $0.5 \mathrm{~T}$; (c) $0.8 \mathrm{~T}$; (d) $1.0 \mathrm{~T}$. The vorticity value ranges from -90 to 90 with intervals of 10 , solid and dashed lines denote positive and negative contours.
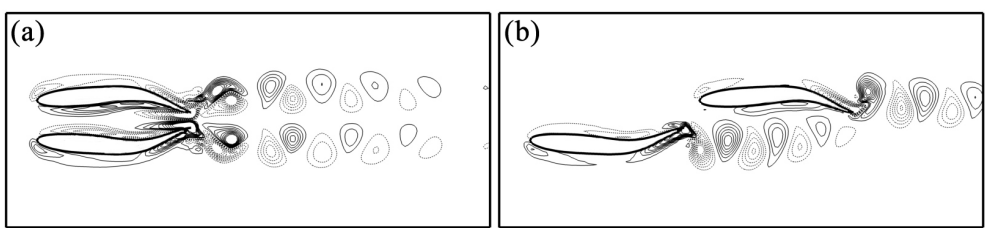

Figure 7. Vorticity contours in the anti-phase case of (a) $D_{x}=0.0, D_{y}=0.3 ;(b) D_{x}=1.1$, $D_{y}=0.3$. The vorticity value ranges from -90 to 90 with intervals of 10 , solid and dashed lines denote positive and negative contours. 
so as the propulsive efficiency (except the case of $\mathrm{D}_{\mathrm{x}}=0.25, \mathrm{D}_{\mathrm{y}}=0.3$ which is similar to a single one), although the increased power is required. The best performance is obtained by two paratactic hydrofoils $\left(D_{x}=0.0\right)$, and no better performance than that of a single hydrofoil is achieved by the anti-phase system when $\mathrm{D}_{\mathrm{x}} \geq 0.5$.

\section{Conclusion}

In the present work, the flow over two wiggling hydrofoils in diagonal formation has been simulated using the immersed boundary method, both the in-phase and the anti-phase wiggling motions are investigated. It is found that, whether the hydrodynamic beneficent obtained depends on the longitudinal spacing between individuals, when the longitudinal spacing is smaller than body length $\left(D_{x}\right.$ $<1.0$ ), the downstream hydrofoil has the increased thrust and efficiency while the upstream hydrofoil is dragged, when the longitudinal spacing is larger than body length $\left(D_{x}>1.0\right)$, the upstream hydrofoil obtains the increased thrust and efficiency but the downstream is dragged. For the whole two-hydrofoil system, if wiggles in-phase, the best performance of the largest thrust coefficient and propulsive efficiency is gained when the longitudinal spacing equals to half of the body length $\left(D_{x}=0.5\right)$, but if wiggles anti-phase, the paratactic formation is beneficial to obtain the highest thrust coefficient and propulsive efficiency. The results obtained here may be helpful to understand the hydrodynamics in fish school, although the two dimensional model used in this study is simplified, and the three dimensional model will be made in the further work.

\section{Acknowledgements}

This work is supported by the National Natural Science Foundation of China (grant number 11462015) and the Aeronautical Science Foundation of China (grant number 2015ZC56007).

\section{References}

[1] Weihs, D. (1973) Hydromechanics of Fish Schooling. Nature, 241, 290-291. https://doi.org/10.1038/241290a0

[2] Liao, J.C. (2007) A Review of Fish Swimming Mechanics and Behaviour in Altered Flows. Philosophical Transaction Royal Society of London. Series B, Biological Sciences, 362, 1973-1993. https://doi.org/10.1098/rstb.2007.2082

[3] Liao, J.C., Beal, D.N., Lauder, G.V. and Triantafyllou, M.S. (2003) Fish Exploiting Vortices Decrease Muscle activity. Science, 302, 1566-1569. https://doi.org/10.1126/science.1088295

[4] Beal, D.N., Hover, F.S., Triantafyllou, M.S., Liao, J.C. and Lauder, G.V. (2006) Passive Propulsion in Vortex Wakes. Journal of Fluid Mechanics, 549, 385-402. https://doi.org/10.1017/S0022112005007925

[5] Zhang, J., Childress, S., Libchaber, A. and Shelley, M. (2000) Flexible Filaments in a Flowing Soap Film as a Model Forone-Dimensional Flags in a Two-Dimensional Wind. Nature, 408, 835-839. https://doi.org/10.1038/35048530

[6] Zhu, L. and Peskin, C.S. (2003) Interaction of Two Flapping Filaments in a Flowing 
Soap Film. Physics of Fluids, 15, 1954-1960. https://doi.org/10.1063/1.1582476

[7] Shelley, M.J. and Zhang, J. (2011) Flapping and Bending Bodies Interacting with Fluid Flows. Annual Review of Fluid Mechanics, 43, 449-465.

https://doi.org/10.1146/annurev-fluid-121108-145456

[8] Ristroph, L. and Zhang, J. (2008) Anomalous Hydrodynamic Drafting of Interacting Flapping Flags. Physical Review Letters, 101, 194502. https://doi.org/10.1103/PhysRevLett.101.194502

[9] Jia, L.B. and Yin, X.Z. (2008) Passive Oscillations of Two Tandem Flexible Filaments in a Flowing Soap Film. Physical Review Letters, 100, 228104. https://doi.org/10.1103/PhysRevLett.100.228104

[10] Favier, J., Revell, A. and Pinelli, A. (2015) Numerical Study of Flapping Filaments in a Uniform Fluid Flow. Journal of Fluids and Structures, 53, 26-35. https://doi.org/10.1016/j.jfluidstructs.2014.11.010

[11] Khalid, M.S.U., Akhtar, I. and Dong, H. (2016) Hydrodynamics of a Tandem Fish School with Asynchronous Undulation of Individuals. Journal of Fluids and Structures, 66, 19-35. https://doi.org/10.1016/j.jfluidstructs.2016.07.008

[12] Deng, J., Shao, X.-M. and Yu, Z.-S. (2007) Hydrodynamic Studies on Two Traveling Wavy Foils in Tandem Arrangement. Physics of Fluids, 19, 113104. https://doi.org/10.1063/1.2814259

[13] Dong, G.-J. and Lu, X.-Y. (2007) Characteristics of Flow over Traveling Wavy Foils in a Side-by-Side Arrangement. Physics of Fluids, 19, 057107. https://doi.org/10.1063/1.2736083

[14] Su, S.-W., Lai, M.-C. and Lin, C.-A. (2007) An Immersed Boundary Technique for Simulating Complex Flows with Rigid Boundary. Computers \& Fluids, 36, 313-324. https://doi.org/10.1016/j.compfluid.2005.09.004

[15] He, G.-Y., Zhang, S.-G. and Zhang, X. (2010) Thrust Generation and Wake Structure of Wiggling Hydrofoil. Applied Mathematics and Mechanics, 31, 585-592. https://doi.org/10.1007/s10483-010-0506-9

\section{Submit or recommend next manuscript to SCIRP and we will provide best service for you:}

Accepting pre-submission inquiries through Email, Facebook, LinkedIn, Twitter, etc. A wide selection of journals (inclusive of 9 subjects, more than 200 journals) Providing 24-hour high-quality service

User-friendly online submission system

Fair and swift peer-review system

Efficient typesetting and proofreading procedure

Display of the result of downloads and visits, as well as the number of cited articles

Maximum dissemination of your research work

Submit your manuscript at: http://papersubmission.scirp.org/

Or contact jamp@scirp.org 